\title{
EL PROCESAMIENTO DE PALABRAS CON CONTENIDO EMOCIONAL EN PERSONAS TIIPICAS Y EN AFÁSICOS
}

\author{
EMOTIONAL WORDS PROCESSING IN TYPICAL
} AND APHASIC PEOPLE

\section{CARLOS J. ÁLVAREZ}

Universidad de La Laguna. La Laguna, España.

calvarez@ull.es

\section{JANETH HERNÁNDEZ-JARAMILLO}

Universidad del Rosario. Bogotá, Colombia.

blanca.hernandez@urosario.edu.co

\section{RESUMEN}

Este artículo aporta elementos teóricos y empíricos para entender el procesamiento de la información emocional verbal. Se incorpora evidencia que proviene de sujetos sanos y, de manera importante, de personas con daño cerebral, como quiera que los resultados en pacientes con afasia permiten la contrastación de los modelos de procesamiento cognitivolingüístico. Se exploran las diferencias en el procesamiento de palabras emocionales en función de la localización hemisférica, el género (hombres-mujeres), desempeño de sujetos "sanos" versus personas con daño cerebral, y valencia emocional de las palabras, entre otras. En su conjunto, la evidencia señala al menos que 1) hay un patrón similar en personas sanas y pacientes con afasias sobre la estrategia de lectura específica de palabras emocionales por el hemisferio derecho, 2) existe un procesamiento temprano de la información emocional, 3) el procesamiento del lenguaje de hombres y mujeres bajo ciertas circunstancias puede ser diferente, 4) la información emocional está dominada por el hemisferio derecho, en particular aquélla con connotación negativa y 5) hay un efecto de facilitación de las palabras con connotación negativa, en comparación con aquéllas positivas y neutras.

Palabras clave: Palabras emocionales, afasia, lenguaje, valencia emocional.

\section{ABSTRACT}

The purpose of this paper is to provide theoretical and empirical understanding about of the processing of verbal emotional information. It incorporates evidence from healthy 
subjects, and importantly, people with brain damage, whatever the outcome in patients with aphasia allow the testing of models of cognitive-linguistic processing. We explore differences in the processing of emotional words according to the hemispheric location, gender (male-female), performance of "healthy" subjects versus people with brain damage and emotional valence of words, among others. Taken together, the evidence points to less that 1) there is a similar pattern in healthy individuals and patients with aphasia on specific reading strategy of emotional words by the right hemisphere, 2) there is an early processing of emotional information, 3) processing the language of men and women under certain circumstances may be different, 4) emotional information is dominated by the right hemisphere, particularly those with negative connotation and 5) there is an effect of facilitating the words with negative connotations, compared with those positive and neutral.

Keywords: Emotional words, aphasia, language, emotional valence.

Recibido: 30.05.2012. Aceptado: 03.09.2012.

\section{INTRODUCCIÓN}

T a exploración de la relación entre los aspectos lingüísticos y extralingüísticos del comportamiento comunicativo de las personas con alteraciones neurogénicas del lenguaje oral y/o escrito, denominadas afasias, provee elementos para comprender la comunicación afectiva en el contexto pragmático. La conciencia general sobre el contexto social y la conciencia específica del interlocutor y su estatus mental o contenido emocional son factores que contribuyen al éxito comunicativo (Lesser y Milroy, 1993). La comunicación de la emoción es un asunto multidimensional, involucra varios y diversos modos o canales simultáneamente -expresión facial, gestos, postura, prosodia, voz, y el propio lenguaje-; se ha sugerido que estas diferentes formas de expresión emocional podrían corresponder también a distintos sistemas neuronales, tal como se observa en pacientes con daños cerebrales selectivos (Borod, 1993).

Los diferentes flujos de activación cortical y del procesamiento de la información en los hemisferios derecho e izquierdo pueden ser el resultado de un número de rasgos distintivos en el procesamiento de señales prosódicas, visuales y verbales, las cuales a la larga determinan la comprensión sobre la connotación, el afecto y los aspectos pragmáticos de la comunicación. La comprensión de la información emocional ha sido tradicionalmente atribuida al procesamiento del hemisferio derecho. En consecuencia, la investigación sobre la ejecución de pacientes afásicos con daño del hemisferio izquierdo (DHI) típicamente se centra en aspectos meramente lingüísticos, mientras que la evidencia sobre personas con lesión neurológica que compromete el hemisferio derecho (DHD) enfatiza la respuesta al contenido emocional o al contexto pragmático. 
Ha habido un especial interés por otros aspectos comunicativos del comportamiento afectivo que co-ocurren con el lenguaje, tales como la expresión facial, el movimiento corporal y los cambios en la voz. Sin embargo, las consideraciones emocionales del lenguaje oral se registran tempranamente en la historia de la afasiología. Broca fue quizás el primero en observar que las personas con afasia pueden conservar la habilidad para expresar ciertos aspectos emocionales y automáticos del habla. En 1861 había notado cómo algunos pacientes, bajo ciertas circunstancias, eran capaces de modificar la forma de expresar palabras con connotación emocional diferente (Fancher, 1990).

La conservación de la experiencia emocional en personas con afasia ha sido puesta a prueba desde principios del siglo XX (Pierre Marie, 1906, citado por Lorch, Borod y Koff, 1998). En general, este tipo de evidencia permite señalar que los pacientes con afasia pueden expresar contenido emocional más que otros tipos de rasgos lingüísticos; en particular, aquellos quienes tienen DHI son exitosos, en tareas que demandan la expresión y percepción facial espontáneas, en cuanto a: 1) la respuesta e intensidad de la expresión emocional facial instintiva, 2) el uso de la expresión facial y de los cambios entonacionales de la voz como canales de expresión emocional, 3) la independencia de los enunciados no verbales, 4) la expresión de la valencia positiva o negativa (agrado o desagrado de un estímulo) de su emocionalidad verbal, 5) la intensidad de la comunicación verbal de lo emocional, 6) el uso del discurso en contextos emocionales, 7) la mediación del contexto emocional sobre aspectos pragmáticos del discurso, 8) la facilitación del contexto emocional sobre la calidad de los movimientos o praxias bucofaciales y 9) la determinación del contexto emocional sobre la intensidad de las expresiones faciales (Lorch et al., 1998). Así las cosas, la comunicación emocional no verbal suele ser mejor en los pacientes con afasia que su competencia verbal. Cabe también reseñar que tanto pacientes con afasia no fluida (dificultad motora o del lenguaje expresivo) como fluida (dificultad sensitiva o de la comprensión del lenguaje) producen más gestos en contextos de conversación espontánea, en comparación con personas sin daño neurológico.

\section{DIFERENCIAS EN LA LOCALIZACIÓN}

En las últimas tres décadas se han llevado a cabo diferentes experimentos que exploran el procesamiento y la producción de palabras emocionales, en personas con y sin compromisos neurológicos (ej., Lorch et al., 1998; Landis, Graves y Goodglass, 1982; Landis, Regard, Graves y Goodglass, 1983; Cicero, Borod, Santschi, Erhan, Obler, Agosti et al., 1999; Borod, 1992; Graves, Landis y Goodglass, 1981; Hamann, 2001; Strauss, 1983). En particular, una revisión conducida por Landis (2006) de una serie de diferentes experimentos usando imágenes de resonancia 
magnética funcional o tomografía por emisión de positrones en estudios sobre el procesamiento de información emocional (palabras y expresiones faciales emocionales) de personas con y sin afasia, concluye que existe una aparente implicación de ambos hemisferios en el procesamiento de palabras emocionales, en niveles diferentes. Las conexiones de la vía estriada de la amígdala izquierda actuarían como un detector del contenido emocional de las palabras en un estadio muy temprano del procesamiento; esta amígdala respondería subsecuentemente modulando la respuesta cortical a las palabras emocionales asimétricamente, registrando menor sensibilidad de la corteza visual izquierda a las palabras emocionales de lo que lo hace el hemisferio derecho (Landis, 2006).

Además de estos hallazgos, se ha reportado un comportamiento diferente de pacientes confrontados a palabras emocionales versus no emocionales. En una tarea, en la cual participaron pacientes afásicos con lesión cerebral unilateral, que implicaba lectura en voz alta y escritura al dictado de estímulos que se correspondían con palabras abstractas emocionales y no emocionales de cuatro letras fueron halladas diferencias entre ambos tipos de estímulos. Cuando se trataba de producir palabras no emocionales, los pacientes demostraban respuestas aleatorias y fallidas; algunas veces, incluso indicaban que conocían el significado de aquello que no podían producir. En contraste, cuando fueron presentadas palabras emocionales, los pacientes con frecuencia sonreían y producían la palabra sin vacilaciones. Las diferencias en el procesamiento de las palabras emocionales en comparación con las no emocionales ratifica la ventaja en el procesamiento de los primeros estímulos sobre los segundos, incluso en tareas de escritura (Landis et al.,1982).

Aunque, a primera vista, parece existir una especialización del hemisferio derecho para las palabras emocionales, es cierto, como se mencionó más arriba, que la evidencia que proviene de estudios usando imágenes de resonancia funcional o tomografía por emisión de positrones (Baas, Aleman y Kahn, 2004) apunta a la participación de ambas amígdalas, en especial de la amígdala izquierda para el caso de las palabras emocionales. Un interesante estudio (Vuilleumier, Richardson, Armony, Driver y Dolan, 2004) en el cual participaron pacientes con lesión de la amígdala, del hipocampo o de ambas regiones, apareados con controles sanos, que fueron expuestos a imágenes de expresiones faciales de miedo y neutras, mostró que el usual incremento en la activación observado en el córtex visual ante estímulos que representaban rostros de espanto en comparación con caras de expresión neutra fue menor en pacientes que presentaban esclerosis de la amígdala. Este hallazgo señala que la amígdala podría regular directamente la percepción emocional vía modulación del input cortical visual; lo cual sugiere, a su vez, la existencia de conexiones visuales extraestriadas paralelas vía amígdala.

Si uno considera que la respuesta a una tarea de decisión léxica, en la cual se debe decidir si el estímulo presentado es o no una palabra, toma aproximadamente entre 350 y 500 milisegundos (ms), deduciendo $200 \mathrm{~ms}$ del tiempo de ejecución 
motora pura, se tendría una ventana temporal de 150 a $300 \mathrm{~ms}$ para el análisis cognitivo-perceptual en su conjunto. En orden de influir en el procesamiento cortical perceptual, un enlace extraestriado paralelo vía la amígdala podría tomar poco tiempo (Landis, 2006). Investigaciones sobre el curso temporal del procesamiento visoperceptual muestran que existe un rápido reconocimiento y categorización de las palabras, cercano a los primeros $150 \mathrm{~ms}$ (ej., Pulvermüller, 2001). Tales modulaciones tempranas en potenciales relacionados con evento (PRE) para palabras versus no palabras reflejan procesos visoperceptuales asociados con tareas de decisión léxica, mientras que las modulaciones tardías expresan análisis sintácticos y semánticos (Friederici, 1997; Lavric, Pizzagalli, Forstmeier y Rippon, 2001). Curiosamente, efectos tempranos de PRE (> 150ms) han sido observados en la atribución de la valencia emocional de las palabras (positiva o negativa por ejemplo: amor versus guerra) (Bernat, Shevrin y Snodgrass, 2001). De la misma forma, respuestas a información emocional facial fueron observadas en potenciales cercanos a los 120ms (Pizagalli, Regards, y Lehmann, 1999; Holmes, Vuilleumier y Eimer, 2003; Pourtois, Grandjean, Sander y Vuilleumier, 2004). Un estudio de Ortigue, Michel, Murray, Mohr, Carbonnel y Landis, reportado el 2004, apoya el rol del hemisferio izquierdo como filtro temprano para las palabras de connotación emocional. Este rápido reconocimiento y categorización de las palabras emocionales es posible independientemente de los análisis posteriores semánticos y sintácticos, pero se traza un reflejo inicial en el hemisferio izquierdo únicamente para las palabras emocionales.

La especialización hemisférica es un hecho casi incontrovertible en pacientes con lesiones unilaterales o desconexión hemisférica, pero no así en sujetos sanos, por razones que aún no son claras. Landis (2006) ofrece una interesante explicación sobre la especialización funcional hemisférica en sujetos sanos. Señala que ello puede depender de estados propios del funcionamiento cerebral en el momento del input de los estímulos; de manera que el uso o no de las diferentes estrategias de cómputo y el destino de procesamiento subsecuente de la información estarían determinando que la información sensorial temprana sea preferentemente transferida y/o procesada por uno u otro hemisferio. No obstante, si se acepta la dependencia del estado de funcionamiento del cerebro en el momento de recepción de los estímulos, los resultados comportamentales pueden variar considerablemente.

La evidencia conduce a pensar que las personas con DHD tienen un mayor déficit para el procesamiento de estímulos emocionales, mientras que aquellos con DHI, aunque también tienen dificultad en tareas emocionales, su ejecución no exhibe diferencias sustanciales en comparación con tareas no emocionales; ello atribuye un rol especial al hemisferio derecho en la percepción de la emoción.

Finalmente, una visión reduccionista del especial rol del hemisferio derecho en la percepción o apreciación de la emoción podría sugerir que ello es así, en tanto 
la emocionalidad involucra estrategias integrativas u holísticas y funciones no verbales para las cuales es sabido que el hemisferio derecho es dominante; de hecho, se reconoce que el hemisferio derecho tiene una gran capacidad de organización e integración multimodal y un alto grado de interconectividad en comparación con el hemisferio izquierdo (Borod, Andelman, Obler, Tweedy y Welkowitz, 1992).

\section{DIFERENCIAS ENTRE HOMBRES Y MUJERES}

Es aceptado que las mujeres son emocionalmente más expresivas que los hombres, y que han desarrollado una mayor habilidad para percibir y entender las expresiones emocionales. En consecuencia, podrían ser más dependientes de este tipo de claves en el procesamiento de la información (Landis, 2006; Schirmer, Kotz y Friederici, 2002).

Uno de los primeros experimentos en reportar diferencias en la lateralización del procesamiento de las palabras en función del género fue conducido por Graves et al. (1981). Para el caso, los hombres, a diferencia de las mujeres, presentaron dos diferentes estrategias de lectura, dependiendo de si las palabras eran presentadas por el campo visual derecho o izquierdo. Los hombres mostraron una fuerte ventaja en las palabras emocionales cuando éstas fueron proyectadas al hemisferio derecho; mientras que las mujeres exhibieron un moderado efecto de ventaja de las palabras emocionales, con independencia del hemisferio sobre el cual eran proyectados los estímulos.

Recientes datos de un estudio empleando resonancia magnética funcional (Cahill, Uncapher, Kilpatrick, Alkire y Turner, 2004) reportan una interacción entre la emocionalidad de los estímulos, la lateralización en la activación de la amígdala y el género. Veintitrés voluntarios sanos (12 hombre; 11 mujeres) debieron ver y clasificar, de acuerdo al nivel de excitabilidad emocional, escenas de diferente valencia emocional del set de estímulos del International Affective Picture System. Dos semanas después, en una prueba incidental de reconocimiento, fue evaluada la memoria sobre los estímulos presentados. Los resultados demostraron una fuerte relación entre la actividad de la amígdala derecha y la memoria para aquellos estímulos juzgados como de alta excitabilidad, más en los hombres que en las mujeres, mientras que en estas últimas hubo una relación significativa entre la actividad de la amígdala izquierda y la memoria para estímulos excitatorios.

En contraste, un metaanálisis de 65 estudios de procesamiento emocional usando neuroimagen publicados entre 1992 y 2002 (Wager, Phan, Liberzon y Taylor, 2003), en el cual se analizó el efecto del tipo de estímulo, el método de inducción de la respuesta emocional y la demanda cognitiva de la tarea sobre la lateralización hemisférica, los resultados no fueron conclusivos; en el sentido que, aunque hubo mayores picos de activación en el hemisferio izquierdo que en el 
derecho (contrario a la hipótesis de superioridad del hemisferio derecho en el procesamiento emocional), éstos no tuvieron significancia estadística. Los hallazgos indican que ciertas regiones del cerebro exhiben lateralización del procesamiento emocional, al igual que en respuesta a la valencia emocional. Fueron hallados los siguientes resultados en relación al procesamiento emocional: 1) lateralización del córtex frontal izquierdo sin diferencias por valencia, 2) lateralización izquierda en la activación de la amígdala en especial para palabras de connotación negativa, 3) lateralización izquierda de la activación del córtex medial prefrontal, 4) lateralización derecha de los ganglios basales para el caso de palabras de carga emocional positiva, 5) activación del cerebelo y la ínsula para emociones negativas, y 6) activación del giro fusiforme y la corteza occipital superior izquierdos como regiones respuesta asociadas). En relación con las respuestas emocionales diferenciales en función del género, el análisis de activación cerebral no mostró mayor activación para el caso del procesamiento emocional en mujeres; de hecho, para el caso de los hombres se registraron mayor número y magnitud de picos en la activación que producían diferentes estímulos emocionales, aunque los patrones de lateralización fueron similares para hombres y mujeres. En resumen, parece no existir un claro efecto del género en la activación de la amígdala, aunque hubo un moderado sesgo de lateralización de la activación de la amígdala izquierda para hombres que para mujeres. En este mismo sentido y en otro estudio, un análisis sobre el efecto del género en tareas de percepción emocional léxica no mostró diferencias en el éxito con el cual hombres y mujeres respondían a estímulos emocionales (Borod et al., 1992).

Una investigación sobre la influencia de aspectos emocionales en la prosodia del habla, es decir, los cambios en los patrones de entonación, modulación, acentuación y ritmo sobre el procesamiento visual de palabras de valencia positiva versus negativa (Schirmer et al., 2002) indica que este tipo de modulaciones prosódicas en el procesamiento de las palabras y su curso temporal difiere en función del género. Se reportan menores intervalos entre el priming prosódico (frases semánticamente neutras producidas con diferencias entonacionales que denotan felicidad o tristeza) y la palabra target en la ejecución de las mujeres; mientras que para los hombres este intervalo fue mayor, aunque se halló un efecto similar de la prosodia emocional sobre el procesamiento de las palabras. Ello indica que las mujeres hacen un uso temprano de los marcadores prosódicos emocionales durante el procesamiento de las palabras en comparación con los hombres. Esta ventaja quizás obedezca a las diferencias tanto en el procesamiento como en la organización del lenguaje en el cerebro (Shaywitz y Shaywitz, 1995). La evidencia que proviene de estudios con resonancia magnética funcional y lateralización visual, algunos de ellos presentados en el apartado anterior, sugieren una fuerte lateralización de la funciones del lenguaje en el hombre en el hemisferio izquierdo, mientras que para las mujeres hay una mayor tendencia a una organización bilateral. Schirmer 
et al. (2002) sugieren que debido a que el procesamiento prosódico emocional es atribuido al hemisferio derecho, las mujeres quizás saquen provecho de las conexiones neuronales cercanas entre las áreas que procesan el contenido semántico del lenguaje y aquellas que resuelven los aspectos prosódicos, relacionados con los aspectos formales de la expresión oral que atribuyen connotaciones diferentes en función de la duración, intensidad, línea melódica y tonalidad del habla. Sumado a la lateralización, la conectividad interhemisférica podría ser responsable de la rápida interacción entre la carga prosódica y la información verbal en las mujeres, dado que estas interconexiones facilitan la transferencia de la información entre los dos hemisferios. Es posible también que el procesamiento del lenguaje en sí mismo sea diferente en hombres y mujeres; de manera que, dependiendo del género, los distintos tipos de información jueguen un rol a lo largo del procesamiento lingüístico.

En el reporte de Schirmer et al. (2002) también fue hallada una diferencia en el procesamiento de palabras positivas y negativas en el grupo de hombres que en el de mujeres; con un menor tiempo requerido para las palabras positivas que negativas, independientemente del priming prosódico. Ello quizás indique que los hombres procesan las palabras atendiendo a la valencia, y de forma independiente de la información prosódica. Esto sugiere, a su vez, que la combinación de la valencia y la prosodia puede ser procesada más eficientemente por las mujeres que por los hombres. En consecuencia, es posible pensar que esta ventaja de las mujeres se aplique únicamente a situaciones en las cuales las emociones son expresadas en el nivel verbal y prosódico, pero no para el procesamiento verbal solo. Quizás también existan diferencias de género en la expresión emocional, con una preferencia de los hombres en el uso de expresiones emocionales verbales en comparación con las mujeres, quienes tendrían un mayor uso de la prosodia en la expresión de contenido emocional.

Mohr, Michel, Lantz, Ortigue, Viaud-Delmon y Landis (2005) registraron la actividad cerebral de participantes sin antecedentes de enfermedad neurológica (11 mujeres y 11 hombres), quienes ejecutaron una tarea de decisión léxica lateralizada (procesamiento hemisferio izquierdo) sobre palabras tanto de contenido emocional como neutras (procesamiento del hemisferio derecho). Se encontró una ventaja de las palabras emocionales y no hubo dependencia de los estados funcionales cerebrales en las mujeres, mientras que para el caso de los hombres fueron hallados patrones completamente diferentes en relación con dos estados de funcionamiento. Los estados de funcionamiento cerebral se correspondieron a dos tipos de mapeo: uno, con mapas de orientación anterior derecha-posterior izquierda, y otro, con distribución anterior izquierda-posterior derecha. Ello indica que los hombres, dependiendo de su estado funcional cerebral, bien pueden 
usar una estrategia de procesamiento dependiente de la connotación emocional de las palabras, que involucra exclusivamente el hemisferio derecho; o un computo del hemisferio izquierdo, en el cual se asumen las palabras más como palabras que como cargas de contenido emocional. En el citado estudio, esta parece ser la estrategia por la cual optaron las mujeres, independientemente del campo visual de presentación de los estímulos y de los estados funcionales.

Lo anterior, en términos del modelo de Zaidel (1983) sobre el "acceso directo" versus "el relevo calloso" significa que los hombres, bajo un estado funcional cerebral, inducen (left anterior-right posterior orientation maps) una condición de acceso directo, donde los hemisferios tratan la información en su propio estilo de procesamiento, mientras que en otro estado funcional cerebral (right anteriorleft posterior orientation) se induce una condición de "relevo calloso", en la cual la información es procesada por el hemisferio dominante, independientemente del campo visual de presentación. Todo esto sugiere que los estados de funcionamiento cerebral reflejan diferencias de la fuerza inhibitoria o excitatoria de la transmisión del cuerpo calloso. En las mujeres parece, al menos para el estudio de Mohr y colaboradores, activarse únicamente la condición de "relevo calloso". De hecho, la amígdala parece jugar un rol en la transmisión de la información emocional, y podría entonces ser sensible al género y a los estados de funcionamiento cerebral. Hoy es ampliamente aceptado que la amígdala tiene un papel específico en el procesamiento de información relacionada con situaciones atemorizantes, y que es altamente dependiente de la intensidad del estímulo emocional más que de su valencia.

Vale la pena mencionar, más allá de estos interesantes hallazgos, el valor que cobra el contexto en el procesamiento de las palabras, cuya investigación usualmente ha atendido a los efectos de la estructura semántica o de los facilitadores contextuales, demostrados en numerosos estudios usando priming. Estos estudios, en su conjunto, muestran altas tasas de acierto y tiempos de reacción en la respuesta ante palabras emparejadas con frases de contexto relacionadas, en comparación con aquellos estímulos no coincidentes. Incluso los experimentos con potenciales exponen una sensibilidad al efecto del contexto durante el procesamiento de palabras (Kutas y Hillyard, 1980). Sin embargo, el contexto no se limita solo a lo semántico. De hecho, como se ha venido mencionando, las claves no verbales, la prosodia y la expresión facial constituyen recursos de información adicional. En consecuencia, resulta de gran interés el estudio del curso temporal de la interacción entre, por ejemplo, los aspectos prosódicos emocionales y el significado de las palabras, tal como fue abordado en el experimento de Schirmer et al. (2002). Es de esperar que el contexto prosódico emocional module el procesamiento de las palabras, como lo hace el contexto semántico. 


\section{DIFERENCIAS ENTRE SUJETOS SANOS Y AFÁSICOS}

La evidencia sobre la dominancia del hemisferio derecho en el procesamiento de información emocional ha sido obtenida a partir de experimentos usando tiempos de reacción y estudios de mapeo cerebral en personas sanas. Este tipo de investigación está de acuerdo en atribuir la percepción y la emocionalidad en sí mismas al hemisferio derecho, perspectiva teórica que se conoce como "hipótesis cualitativa" (Hielscher, 2004). En contraste, la "hipótesis cuantitativa" que proviene de estudios en cerebros lesionados parece converger en la dominancia del hemisferio izquierdo para las emociones positivas y del derecho para aquéllas de connotación negativa (Goldstein, 1939). Así, por ejemplo, se ha encontrado una notable incidencia de estados depresivos en pacientes con lesión cortical izquierda (Robinson, Arndt, Starkstein, Forrester y Geisler, 1993). Algunos otros reportes de mapeo cerebral y electrofisiología apoyan la idea de la dominancia derecha en la percepción de estímulos negativos (Canli, Desmond, Zhao Glover y Gabrieli, 1998; Duda y Brown, 1984). Aun así, existe una tercera perspectiva sobre la dominancia emocional. Ross, Homan y Buck (1994) presentan evidencia empírica sobre la especialización del hemisferio derecho en el procesamiento de la emoción básica, mientras postulan una dominancia del hemisferio izquierdo para la emoción social. El desempeño de pacientes con DHD en la comprensión de textos muestra una relativa pobreza en la ejecución de tareas de categorización emocional comparada con decisiones basadas en información más específica, donde las situaciones fueron descritas acorde con procesos de apreciación emocional y teorías de atribución causal. Varios textos que atienden a elaboraciones teóricas sobre el control central de la emoción coinciden en que las emociones básicas son desarrolladas tempranamente, y suelen estar asociadas a situaciones ordinarias o con claves verbales, no verbales o contextuales simples. Sin embargo, la emoción social aparece tardíamente, se vincula con situaciones de interacción más complejas y requiere un profundo procesamiento conceptual, mediado usualmente por el lenguaje y, en consecuencia, por el hemisferio izquierdo (Ross, Homan y Buck, 1994).

Hay también una tendencia en señalar que el hemisferio derecho está involucrado en tareas verbales complejas, como la interpretación del lenguaje metafórico, el humor, los actos de habla indirectos, la solución de ambigüedad léxica y, desde luego, la comprensión de textos. Por ejemplo, para la realización de inferencias en textos, parece que se da una activación temprana del hemisferio derecho; en contraste, el hemisferio izquierdo estaría involucrado, más tardíamente, en procesos relacionados con el establecimiento de la coherencia y con la comprensión de las inferencias. Por ello, como ya se mencionó, las inferencias emocionales podrían ser especialmente tareas de procesamiento difíciles para pacientes DHD, optando por compensaciones del hemisferio izquierdo (Beeman, Bowden y Gernsbacher, 2000). 
Un estudio sobre el desempeño en individuos sanos (Graves, Landis y Goodglass, 1981) indica que los hemisferios derecho e izquierdo juegan un rol diferente en las estrategias de lectura de palabras emocionales y no emocionales. La presentación de palabras en el campo visual derecho (hemisferio izquierdo-dominante) resulta en una ejecución superior para el reconocimiento de palabras; aunque no parece haber diferencias entre palabras emocionales y no emocionales. Sin embargo, la presentación de las palabras en el campo visual izquierdo (hemisferio derecho-no dominante) mostró un aleatorio reconocimiento de las palabras no emocionales, pero un muy buen reconocimiento de las palabras emocionales. Según los autores, ello podría sugerir que en los cerebros sanos pueden activarse dos diferentes estrategias de lectura: una estrategia del hemisferio izquierdo, la cual es generalmente buena en la lectura sin atender a diferencias entre palabras emocionales y no emocionales; y una estrategia del hemisferio derecho, de forma que este hemisferio fuese analfabeto para las palabras no emocionales pero casi tan bueno como el hemisferio dominante para procesar palabras de contenido emocional. Otros estudios confirman una ventaja general en el procesamiento de palabras emocionales sobre palabras de contenido neutro (ej., Strauss, 1983; Goodglass, Graves y Landis, 1980).

Goodglass et al. (1980) compararon la ejecución de individuos sanos con el número de palabras que fueron leídas por pacientes con afasia, sobre los campos visuales derecho e izquierdo, y hallaron que los puntajes de los pacientes con afasia estaban muy correlacionados con la ejecución de los participantes sanos en la tarea de decisión léxica lateralizada para el campo visual izquierdo. Sin embargo, no hubo una correlación significativa para el campo visual derecho. Ello sugiere que hay una familiaridad para las palabras de connotación emocional tanto en personas afásicas con DHI como en el procesamiento del hemisferio derecho en sujetos sanos.

Un estudio que compara los resultados de la ejecución de pacientes con DHD versus izquierdo en tareas no verbales, verbales y de percepción emocional (Hielscher, 2004) usando interferencia emocional en comprensión de textos mostró un déficit similar en ambos grupos tanto para tareas verbales como para no verbales. Los participantes (21 con DHI, 26 con DHD y 27 controles) debieron leer una serie de textos, divididos en cuatro situaciones emocionales diferentes (tristeza, culpa, consuelo, orgullo) que incluía inferencias emocionales implícitas. Las tres condiciones experimentales fueron: 1) de coherencia con la emoción que suscitaba la situación de contenido del texto, 2) la similitud emocional con la valencia pero con equivocada atribución causal y 3) valencia desacertada. El análisis de datos, basado en los tiempos de lectura y los aciertos en las tareas de inferencia emocional, permite concluir que existe una inferencia emocional poco significativa tanto en pacientes con DHI como DHD frente a los controles. Solo un efecto del priming del contexto típico emocional fue hallado para las palabras que mostraban 
coherencia emocional en contraste con aquéllas de valencia opuesta. Los participantes con DHI parecieron no sacar provecho del contenido emocional positivo o negativo de la información inferida. Estos mismos pacientes presentaron un ligero efecto de la condición de coherencia emocional para todas las ejecuciones; mientras que aquéllos con DHD sólo exhibieron un efecto en las tareas de decisión léxica que para la lectura de textos. Ello va en consonancia con los resultados reportados por Beeman, Bowden y Gernsbacher (2000) que demuestran una dominancia del hemisferio derecho para procesos tempranos de priming predictivo. Ambos grupos tuvieron una ejecución similar en la categorización de palabras emocionales, expresiones faciales y rasgos prosódicos emocionales.

Quizás la colección más extensa de datos de investigación de pacientes con daño cerebral sobre la organización de la expresión emocional sea la publicada por Joan C. Borod y colaboradores (ej., Borod, Caron y Koff, 1981; Borod, Koff y White, 1983; Borod y Koff, 1984; Borod, Koff, Lorch y Nicholas, 1985, 1986a, 1986b; Borod, Lorch, Koff y Nicholas, 1987; Borod, Koff, Lorch, Nicholas y Welkowitz, 1988; Borod, 1992). Los resultados de estos estudios han sido analizados teniendo en cuenta las diferencias de ejecución en un amplio rango de tareas sobre la emoción de pacientes con DHD, DHI y personas sanas. La hipótesis inicial asumía que las lesiones corticales focales derechas podrían inducir un daño en la percepción y la expresión emocionales.

Lorch, Borod y Koff publicaron en 1998 una revisión de estos y otros datos con el fin de responder a dos interrogantes: uno, qué habilidades de la expresión emocional se mostraban intactas en la ejecución de pacientes con DHI y dos, si estas habilidades diferían del desempeño de sujetos sanos. El objetivo consistió en identificar elementos extralingüísticos preservados en la ejecución de los pacientes para compensar su comunicación. Se realizaron análisis en función de la expresión facial emocional espontánea (si ocurría una respuesta o no), la intensidad de la misma (en una escala de 7 puntos 1= Mínima intensidad a 7= Máxima intensidad) y si la respuesta era apropiada o no, además del canal de comunicación empleado (facial, entonacional o verbal), la contribución proposicional o no proposicional a la comunicación hablada y la verbalización de la emoción. Los puntajes promedio revelaron una ligera mayor respuesta de los pacientes con DHI en comparación con los controles; y ambos grupos se mostraron más receptivos que aquéllos con DHD. En relación con la intensidad de las respuestas, aquéllos con DHI produjeron respuestas más intensas con expresiones faciales marcadas que los controles y que los pacientes con DHD. Sólo fue hallada una correlación significativa entre respuesta e intensidad para el grupo de pacientes con DHI. La contribución de los canales de comunicación facial y entonacional fue mayor en el grupo de personas con DHI que para aquéllos con lesiones derechas y controles. Cuando se compararon las lesiones frontales y no frontales se registró un mayor uso de la expresión facial por los pacientes con lesiones frontales izquierdas, y no frontales derechas 
e izquierdas que por el grupo de pacientes con daño frontal derecho. Ello no es sorprendente, por cuanto es frecuente observar clínicamente, como se mencionó al inicio, que el uso de canales de comunicación no verbal puede suplir o compensar los déficits en la producción de lenguaje espontáneo. Por ello, no resulta impensable que las personas con daño frontal izquierdo o afasias no fluidas usen con menor frecuencia estos canales de lo que lo hacen los otros grupos. Aun así, sí resulta notable que aquéllos con DHI usen la entonación más que los pacientes con lesiones derechas. El análisis sobre la contribución del habla proposicional y no proposicional en las respuestas verbales a la emoción, reveló mayor habla no proposicional en pacientes con $\mathrm{DHI}$ que la de los sujetos control, mientras que en aquéllos con DHD no hubo contribución del habla no proposicional. Finalmente, en el análisis del contenido lingüístico de las respuestas verbales a la emoción realizado en función de si el sitio de la lesión era inter o intra hemisférico, se halló que los pacientes con compromiso izquierdo no frontal o posterior producían un gran número de descripciones inapropiadas más que respuestas verbales emocionales.

Un segundo tipo de análisis hecho por Lorch, Borod y Koff en este mismo trabajo (1998), sobre la adecuada categorización de la valencia de los estímulos, puso de manifiesto que los pacientes con DHI fueron menos exitosos que los controles en comunicar en su discurso la categoría emocional, pero discretamente mejores que estos últimos en la expresión verbal de las emociones con valencia negativa. Asimismo, las personas con DHI tuvieron expresiones más intensas en la comunicación verbal que los controles y los pacientes con DHD (Bloom, Borod, Obler y Koff, 1990).

Un estudio publicado por Bloom, Borod, Obler y Gerstman (1992), en el cual se elicitaba tres tipos de narrativa de dibujos con contenido emocional, contenido neutro o procedural y contenido viso-espacial en movimiento (descripción de acción visual), presentados a pacientes con (lesión derecha e izquierda) y sin daño cerebral, permitió concluir que, frente a factores de tipo pragmático, los pacientes con DHI estuvieron significativamente más limitados en cuanto a la especificidad, revisión, reparación y cantidad de información que el grupo control. Sin embargo, no hubo diferencias significativas sobre factores pragmáticos relacionados con especificidad y cantidad de información, revisión y reparación y selección léxica frente a los controles. No obstante, los pacientes con DHI tuvieron menor dificultad en la narrativa emocional que en la viso-espacial y la procedural. En general, los individuos en cada grupo tuvieron un desempeño mejor o igual en la condición con connotación emocional que en la no emocional, apreciación que se mantuvo para pacientes con DHI indistintamente de si se trataba de una lesión anterior (frontal) o posterior (no frontal). Ello se puede traducir en un efecto de facilitación del contexto emocional sobre los factores pragmáticos en el caso de lesiones del hemisferio izquierdo. Parece que el material con carga emocional puede 
favorecer el desempeño de las personas con afasia en una amplia variedad de tareas (ej., comprensión auditiva, lectura oral, escritura y repetición).

Lorch, Borod y Koff (1998) ofrecen también la descripción de un estudio cuya hipótesis se cifra en saber si las claves emocionales podrían mejorar la ejecución de pacientes apráxicos al proveer una mayor automaticidad o bien una facilitación por contexto emocional. De hecho, si se acepta que el hemisferio derecho está implicado en la expresión de la emoción, es muy posible que las claves emocionales faciliten la respuesta práxica en pacientes con DHI. Como era de esperar, los participantes con lesión izquierda demostraron una importante restricción en las praxias bucofaciales ante tareas con estímulos neutros en relación con los controles y aquéllos con DHD. No obstante, las ejecuciones motoras del primer grupo (DHI) se vieron más facilitadas por claves emocionales adicionales en comparación con los otros dos grupos. Un análisis sobre la expresión emocional voluntaria señaló que la intensidad de la expresión emocional positiva fue mayor para los pacientes con DHI que para los pacientes con DHD y el grupo control; mientras que la valencia positiva fue más intensa que la negativa para las personas con DHI y los controles, sin diferencias estadísticas para el grupo con DHD. En el ámbito terapéutico, se ha demostrado el efecto de facilitación de la información emocional en las apraxias del habla, en la cual se usan otros aspectos del comportamiento cognitivo atribuidos al hemisferio derecho. Ejemplos de ello son la "terapia de entonación melódica", la "terapia de acción visual" y "comunicación visual”.

Finalmente, un estudio que examinó la contribución del canal léxico verbal al procesamiento emocional (Borod, Andelman, Obler, Tweedy y Welkowitz, 1992) involucró a 48 participantes con y sin antecedente de ataque cerebro-vascular, distribuidos en igual número en tres grupos (pacientes con DHI, DHD y controles). Debieron desarrollar tareas de percepción emocional y tareas de control sin contenido emocional, y los resultados sugieren un rol dominante del hemisferio derecho en la percepción de estímulos emocionales basados en rasgos lexicales. Hubo también un efecto de grupo, donde los controles fueron más exitosos que los pacientes con DHD. No obstante, la interacción por grupo por condición modificó estos efectos, y no pareció haber diferencias significativas para el grupo control y el grupo de pacientes con DHI, aunque los sujetos con DHD fueron más exitosos en la ejecución de tareas no emocional que en las de contenido emocional. El análisis de datos sobre las diferencias de grupo por cada tarea indicó que los controles obtuvieron mayores puntajes que los otros dos grupos en ambas tareas (material emocional y no emocional). Aun así, los pacientes DHI fueron más hábiles en tareas emocionales que aquéllos con DHD. Para la identificación de frase hubo también un efecto de grupo, con un mayor éxito para los controles en comparación con otros dos grupos. El examen de las diferentes tareas por cada sujeto y grupo de participantes no señaló diferencias para los controles y aquéllos con DHI. El grupo de pacientes con DHD tuvo un mejor desempeño en tareas no 
emocionales. Las diferencias de grupo por cada tarea indican que el grupo control tuvo mayor éxito en ambas tareas en comparación con los otros grupos. Además, el grupo con DHD fue significativamente más hábil en tareas no emocionales que aquéllos con DHI. Sobre la tarea de discriminación de palabras se dio un efecto de grupo, siendo los controles mejores que el grupo con DHD. El efecto por condición de nuevo señala superioridad en las tareas no emocionales. Además, no se encontraron diferencias de tareas para cada sujeto en los grupos para el control o el grupo con DHI aunque el grupo con DHD tuvo mejor desempeño en tareas no emocionales. El examen de los datos en función de grupos por cada tarea reveló que los controles y aquéllos con DHI fueron mejores que los pacientes con DHD en tareas emocionales. De la misma forma como se registró para las otras medidas de percepción usadas en este estudio, los controles tuvieron mejor desempeño que los otros dos grupos en tareas no emocionales.

En su conjunto, los datos de varios estudios (ej., Bloom, Borod, Obler y Gerstman 1992; Semenza, Pasini, Zettin, Tonik y Portolan, 1986; Borod, Koff, Lorch y Nicholas, 1985) sugieren que los pacientes con DHD tienen déficit en la percepción emocional frente a una variedad de tareas, relacionadas con la identificación o discriminación y la comprensión de palabras y oraciones, comparados con quienes tienen $\mathrm{DHI}$ y aquéllos sin lesiones neurológicas, tal como también parece evidenciarse en investigaciones con otro tipo de estímulos tales como expresión facial y prosodia.

\section{DIFERENCIAS EN FUNCIÓN DE LA EDAD}

Existen evidencias que apoyan diferencias en edad en el procesamiento de palabras emocionales. Por ejemplo, recientes estudios indican que existe un cambio en cómo las emociones son experimentadas y reguladas en la adultez tardía (Carstensen y Charles, 1994; Lawton, Kleban, Rajagopal y Dean, 1992). Los adultos mayores, por ejemplo, reportan niveles altos o al menos similares del afecto positivo y bajos niveles de afecto negativo en comparación con jóvenes o adultos de mediana edad, al igual que ocurre con el control emocional y la estabilidad del humor (Gross, Carstensen, Patsupathi, Tsai, Skorpen y Hsu, 1997). No obstante, la respuesta a estímulos relacionados con imágenes positivas y negativas es similar entre adultos mayores y adultos jóvenes de la misma forma que ocurre en la habilidad para decodificar información emocional de material verbal. Parece, entonces, que la funcionalidad sobre la emoción se preserva, aun cuando no se incrementa con la edad. En la adultez temprana parece que el individuo prioriza la información para el alcance de sus metas, a fin también de estar más preparado para el futuro. Pero con la edad, las personas van incrementando la conciencia del tiempo restante y se motivan a perseguir metas significativamente más emocionales. A esto se 
le denomina la "teoría de la selectividad socioemocional" (Carstensen y Charles, 1998). También es cierto que las personas mayores recuperan mejor la información emocional que la neutra, e incluso su efecto es superior que para los adultos jóvenes (Carstensen y Charles, 1994).

En una investigación conducida con el fin de evaluar las diferencias en la interacción entre la atención y la emoción en función de la edad (Langley, Rokke, Stark, Saville, Allen y Bagne, 2008), en la cual dos grupos de participantes (jóvenes adultos y adultos mayores) debieron identificar palabras de distinta valencia en una tarea de presentación visual serial rápida, se halló que los adultos mayores mostraron aumento de la identificación tanto de palabras positivas como negativas, en comparación con las neutras. Sin embargo, los adultos jóvenes exhibieron un incremento en la identificación de palabras positivas y una reducción en la identificación de palabras negativas. Ello coincide, según los autores, con la idea de que ocurren variaciones en la interacción entre la atención y la emoción con la edad, aunque no hubo evidencia que soportara un cambio emocional consistente con las metas de regulación emocional en un estadio temprano del procesamiento cognitivo. Otro estudio usando también presentación visual serial rápida (Arnell, Killman y Fijavz, 2007) mostró un procesamiento preferencial de palabras emocionales: aquéllas de contenido sexual, especialmente, funcionan como distractores que capturan la atención de forma inconsciente en este tipo de tareas, a expensas de la ejecución en la identificación de las palabras neutras subsecuentes.

En un estudio cuyo propósito fue desarrollar un corpus de palabras emocionales de niños y adolescentes (Neshat-Doost, Moradi, Taghavi, Yule y Dalgleish, 1999), se identificó una creciente habilidad para producir esta clase de palabras en función del grado de escolaridad (primaria y secundaria). Asimismo, los niños mostraron destreza en la categorización de diferentes emociones, al igual que para diferenciar los sentimientos de las cosas que causaban estas emociones. Hubo además una diferencia significativa entre los niños de primaria y secundaria para generar palabras emocionales negativas, no así para las palabras de connotación positiva; ello sugiere una expansión del vocabulario de valor emocional negativo en función del tiempo. Las niñas de escuela secundaria produjeron significativamente más palabras, quizás porque se les atribuye mayores habilidades lingüísticas en comparación con los niños.

\section{DIFERENCIAS EN LA VALENCIA DE LAS PALABRAS}

A partir de la hipótesis de la valencia podría predecirse que las personas con DHD tienen más limitación en el procesamiento de emociones negativas que positivas $y$, en contraste, que quienes tienen DHI encontraran más dificultad con el procesamiento de estímulos positivos. No obstante, algunos estudios muestran 
evidencia contradictoria; por ejemplo, en el citado estudio conducido por Borod et al. (1992) sobre tareas léxicas de percepción emocional no hubo un efecto significativo de la valencia en función de la lateralización de la lesión hemisférica (comparación del hemisferio derecho o izquierdo).

Otro estudio (Vasa, Carlino, London y Min, 2006), con el mismo propósito que el de Nesthat-Doost et al. (1999), de desarrollar un corpus de diferentes tipos de palabras emocionales, éste en particular para ser usado en el análisis de las respuestas cognitivas de niños con desorden de ansiedad, puso de manifiesto que los niños responden selectivamente a las palabras en función del contenido emocional. Vasey, el-Hag y Daleiden (1996), bajo el supuesto de las anormalidades en el procesamiento de la información amenazante en niños con trastornos de ansiedad, mostraron que los niños con este tipo de diagnóstico del comportamiento atienden más a las palabras que expresan amenaza que a aquéllas de tipo neutro. En esta misma vía, se ha señalado también que las mujeres son emocionalmente más reactivas que los hombres (McManis, Bradley, Berg, Cuthbert y Lang, 2001). De hecho, parece que incluso las mujeres producen respuestas emocionales más fuertes para ambos tipos de palabras (positivas y negativas), un resultado consistente con hallazgos de otros estudios en donde se da un aumento de la reactividad psicológica y comportamental en niñas, cuando se compara esta misma asociación en niños. Parece también ocurrir que las palabras amenazantes de base física evocan una respuesta más negativa que aquéllas de base social sin ningún aparente efecto de la edad (Vasey et al., 1996).

Aunque casi todos los estudios sobre valencia se han conducido en monolingües, algunas pocas investigaciones sobre las palabras emocionales han atendido las diferencias en la intensidad del procesamiento en personas bilingües (Ferré, García, Fraga, Sánchez-Casas y Molero, 2010) y han aportado evidencia a la superioridad de las palabras emocionales sobre las no emocionales, con la misma intensidad en la primera y segunda lengua. No obstante, también se ha discutido que la intensidad emocional pudiera experimentarse con mayor fuerza en la lengua materna o primera lengua; por ejemplo, las palabras tabú u obscenas suelen generar menor ansiedad cuando se producen en la segunda lengua (GonzálezReigosa, 1976).

En este mismo sentido, los datos recolectados por Dewaele en una serie larga de reportes (2004a, 2004b, 2006, 2008) revelan que las palabras tabú (maldiciones u obscenas, e incluso las declarativas como "te amo") son mucho más emocionales (tienen una valencia mayor) en la primera lengua. Igual ocurre con aquellas personas que adquieren tardíamente o no tienen suficiencia en una segunda lengua, tienden a producirlas con menor intensidad que como ocurre en su primera lengua. Es de esperarse que si la intensidad de los estímulos emocionales es mayor en la primera lengua se observe una ventaja en la memoria para palabras emocionales cuando son presentadas en la primera lengua, mientras 
que en la segunda este efecto podría no darse o tener una menor magnitud. Ello quizás responda al hecho de que en bilingües tardíos las experiencias emocionales estuvieron asociadas a la primera lengua en su infancia temprana, y probablemente continúen arraigadas hasta la vida adulta, algo diferente a las vivencias emocionales en la segunda lengua. Una más amplia discusión es presentada por Ferré et al. (2010).

Se han reportado otras variables relacionadas con el procesamiento de palabras emocionales en bilingües, además de la dominancia de uno u otro lenguaje o de ambos, entre las que se hallan el contexto y edad de adquisición de la segunda lengua, la similitud entre las lenguas, la normalización de los estímulos, el género y personalidad. Esto a primera vista podría ser muy simplista; sin embargo, hay factores que pueden modular el uso y la percepción de la fuerza emocional de las palabras en la segunda lengua.

Los modelos de procesamiento emocional (Robinson, 1998), y la propia evidencia, proponen que los estímulos relacionados con palabras amenazantes tienen un acceso privilegiado al análisis por el sistema cognitivo (ej., Calvo y Castillo, 2005), y que existe una ventaja en el procesamiento de las palabras de connotación amenazante (Calvo y Eysenck, 2008), quizás debido a que esta clase de palabras o imágenes atraen y capturan la atención, de forma que interfieren en el procesamiento de otro tipo de estímulos. Resulta interesante que este efecto ocurre tempranamente en el procesamiento, de manera que la atención capturada por estímulos emocionales en la visión foveal es muy rápida (Harris y Pashler, 2004). Por ello es esperable que las palabras con carga emocional negativa, presentadas de forma previa, disminuyan y lentifiquen la ejecución en una tarea concurrente. Asimismo, se han señalado mayores tiempos de reacción en respuesta a palabras neutras que para positivas y aún mayor para los estímulos negativos (Vö, Jacobs y Conrad, 2006). Calvo y Castillo discuten también como hallazgo en su estudio la ausencia de efecto de interferencia de la visión parafoveal de palabras emocionales, como lo indica otro estudio sobre la duración de las fijaciones visuales cercanas a las palabras emocionales durante la lectura continua de un texto (2005). Así las cosas, lo que la investigación de Calvo y Castillo advierte es que las palabras con connotación negativa, o de amenaza, no parecen procesarse prioritariamente en la visión parafoveal o tener algún tipo de estadio preatencional; el foco atencional para este tipo de estímulos sólo ocurriría en la visión foveal, es decir, que las palabras amenazantes en la visión foveal hacen interferencia con el procesamiento subsecuente de palabras neutrales. Aun así, en otra investigación Calvo y Eysenck (2008) revelaron un efecto del priming parafoveal, lo que se traduce en la posibilidad que las palabras negativas o amenazantes sean procesadas por la atención encubierta en ausencia del procesamiento de la atención manifiesta, lo que atribuye a esta clase de estímulos un privilegio en el acceso al sistema cognitivo. Hay en estos hallazgos un rol importante de la familiaridad de 
las palabras, por cuanto cuando se controló esta variable, la ventaja sobre el priming de palabras amenazantes no fue significativa. En otro de sus estudios, Calvo y Castillo (2005) dejaron ver la existencia de un efecto de facilitación en los tiempos de respuesta en una tarea de decisión léxica cuando el priming parafoveal se trataba de una palabra amenazante idéntica al estímulo, en comparación con priming no relacionados. A su vez, se aumentaba el efecto en un contexto de congruencia emocional no placentera, contrario a lo ocurrido con palabras positivas en contextos agradables con priming no congruente, quizás porque la condición de incongruencia actúa como distractor o hace interferencia en el momento de respuesta. Esto sugiere un procesamiento paralelo de las palabras con carga emocional amenazante fuera del foco de atención, que coincide, tal como se mencionó arriba, con los modelos de procesamiento emocional frente al automatismo del significado afectivo del input perceptual, en especial al tratarse de estímulos amenazantes. Otras investigaciones también han apuntado a la existencia de una ventaja del priming del campo visual derecho en el reconocimiento de palabras (Ortells, Tudela, Noguera y Abad, 1998; Mondor y Bryden, 1992), que se puede explicar por una asimetría del span perceptual en la lectura (mayor extensión de las fijaciones oculares en el campo derecho que en el izquierdo (Rayner, 1998) o bien por una dominancia del hemisferio izquierdo en el reconocimiento visual de palabras, con lo cual el reconocimiento de palabras es significativamente más exitoso por mecanismos neuronales localizados en el hemisferio izquierdo (Fuentes y Santiago, 1999).

Otro interesante argumento sobre la superioridad del procesamiento de palabras con valencia negativa es la apreciación afectiva, que explicaría la detección parafoveal de las palabras amenazantes, e incluso para el procesamiento paralelo (foveal y parafoveal). Desde luego que ambos tipos de carga emocional son importantes para procesos adaptativos, pero se ha enfatizado la prioridad de los estímulos de aversión. De hecho, aunque los datos son escasos, el tipo de representación (significado) del priming puede modificar el procesamiento parafoveal de las palabras, esto es, el priming semántico supondría una preferencia de unos estímulos frente a otros en función de su significado, por ejemplo si se trata de información de importancia adaptativa. Otros sesgos del efecto priming, en el procesamiento de palabras emocionales, como la similitud ortográfica/fonológica han sido discutidas en varias publicaciones (Abad, Noguera y Ortells, 2003; Lavie y Fox, 2000; MacLeord, Chiappe y Fox, 2002).

Finalmente, el efecto de congruencia en el humor, no gratificante o desagradable, como se mostró a propósito de la investigación de Calvo, Castillo y Fuentes (2006) se incrementa en el priming parafoveal de las palabras con congruencia emocional. Este efecto de asimetría del contenido y el contexto positivo y negativo podría explicarse porque el priming parafoveal involucra una percepción visual automática y, por tanto, sería insensible a los efectos de estímulos de carga 
positiva, en contraposición con aquéllos de contenido emocional negativo. Se ha advertido que el afecto positivo influye en tareas que muestran una alta dependencia de recursos de control cognitivo o que involucran la memoria de trabajo (ej., creatividad, resolución de problemas). Sin embargo, no hay evidencia de que el afecto positivo esté relacionado con la ejecución de tareas que demandan procesos automáticos, tal como la percepción visual.

Este asunto sobre si las palabras emocionales son preprocesadas en la periferia del campo (visión parafoveal) o sin fijación visual se enmarca en el interés de un amplio número de estudios que indagan sobre la información mínima o suficiente que se requiere para identificar una palabra, en especial, si esta información es posible obtener en la atención encubierta (Calvo et al., 2006).

En suma, han sido planteadas varias posibles explicaciones a los hallazgos contradictorios que han emergido en los estudios sobre la valencia y la propia lateralidad. Borod et al. (1992) ofrecen tres de esos razonamientos: 1) Que el modo de procesamiento bajo observación (ej., expresión, percepción, experiencia) afecte el patrón de respuesta, puesto que hay evidencia en relación a que la valencia es una variable más robusta en tareas de expresión y experiencia que de percepción (ej., Hirschman y Safer, 1982). De hecho, se ha especulado sobre la diferencia de especialización hemisférica para la expresión y la experiencia en función de la valencia, y una particular sensibilidad del hemisferio derecho para tareas de percepción en cuanto a la valencia. 2) Que el efecto de la valencia opere bajo un estado de humor determinado, de forma tal que aunque el hemisferio derecho esté implicado en el procesamiento de la valencia es más común que lo haga cuando está involucrado el procesamiento no verbal y/o la comunicación social (Borod et al., (1992). Y 3) Que las diferencias de lateralización en función de la valencia estén afectadas por el género (ver en el apartado sobre diferencias por género Schirmer, Kotz y Friederici, 2002).

\section{CONCLUSIONES}

De todos los trabajos empíricos provenientes de distintas líneas de investigación anteriormente expuestos, es posible extraer una serie de conclusiones teóricas de gran relevancia explicativa sobre el procesamiento de palabras con contenido emocional. A continuación se exponen, de forma genérica, aquellas que resultan más interesantes pero que, sin duda, no son las únicas:

1) hay un patrón similar en personas sanas y pacientes con afasias sobre la estrategia de lectura específica de palabras emocionales por el hemisferio derecho, 2) existe un procesamiento temprano de la información emocional (100-150ms),

3) ciertas regiones del cerebro exhiben lateralización del procesamiento emocional 
y de la respuesta a la valencia emocional; en especial para estímulos visuales, se sugiere la participación de la amígdala izquierda, 4) el procesamiento del lenguaje de hombres y mujeres bajo ciertas circunstancias puede ser diferente, 5) la información emocional está dominada por el hemisferio derecho, en particular aquélla con connotación negativa, 6) el uso de canales de comunicación no verbal puede suplir o compensar los déficits en la producción de lenguaje espontáneo, 7) se da una facilitación del contexto emocional sobre los factores pragmáticos en personas con daño del hemisferio izquierdo, 8) existe un rol dominante del hemisferio derecho en la percepción de estímulos emocionales con base léxica, 9) hay un efecto de facilitación de las palabras con connotación negativa, en comparación con aquéllas positivas y neutras, 10) la existencia de un procesamiento paralelo de las palabras con contenido amenazante fuera (parafoveal) del foco de atención y 11) que la carga emocional de las palabras podría tener la misma intensidad en personas bilingües, aunque ello podría variar en función de la suficiencia, similitud, edad y contexto de adquisición de la segunda lengua.

\section{REFERENCIAS}

Abad, M.J., Noguera, C. y Ortells, J.J. (2003). Influence of prime-target relationship on semantic priming effects from words in a lexical-decision task. Acta Psychologica, 113, 283-295.

Arnell, K.M., Killman, K.V. y Fijavz, D. (2007). Blinded by emotion: Target misses follow attention capture by arousing distractors in RSVP. Emotion, 7, 465477.

Baas, A., Aleman, D. y Kahn, R.S. (2004). Lateralization of amygdala activation. Brain Res, 45, 96-103.

Beeman, M., Bowden, E.M. y Gernsbacher, M.A. (2000). Right and left hemisphere cooperation for drawing predictive and coherence inferences during normal story comprehension. Brain y Language, 71, 310-336.

Bernat, E., Shevrin, H. y Snodgrass, M. (2001). Subliminal visual oddball stimuli evoke a P300 component. Clin. Neurophysiol, 112, 159-171.

Bloom, R.L., Borod, J., Obler, L. y Gerstman, L. (1992). Impact of emotional content on discourse production in patients with unilateral brain damage. Brain and Language, 42, 153-164.

Bloom, R.L., Borod, J.C., Obler, L.K. y Koff, E. (1990). A preliminary characterization of lexical emotional expression in right and left brain-damaged patients. Internationat. Journal of Neuroscience, 55, 71-80.

Bloom, R.L., Borod, J.C., Obler, L. K., Loraine, K. y Gerstman, L.J. (1993). Supression and facilitation of pragmatic performance: Effects of emotional 
content on discourse following right and left brain damage. Journal of Speech Language and Hearing Research, 36, 1227-1235.

Borod, J.C. (1992). Interhemispheric and intrahemispheric control of emotion: A focus on unilateral brain damage. Journal of Consulting and Clinical Psychology, 60(3), 339-348.

Borod, J.C. (1993). Emotion and the brain- Anatomy and theory. An introduction to the special section. Neuropsychology, 7, 427-432.

Borod, J.C., Andelman, F., Obler, L., Tweedy, J. y Welkowitz, J. (1992). Right hemisphere specialization for the identification of emotional words and sentences: Evidence from stroke patients. Neuropsychologia, 30(9), 827-844.

Borod, J.C., Caron, H.S. y Koff, E. (1981). Asymmetry of facial expression related to handedness, footedness, and eyedness: A quantitative study. Cortex, 17, 381-390.

Borod, J.C., y Koff, E. (1984). Asymmetries of affective facial expression. En N. Foxand y R. Davidson (eds.), The psychobiology of affective development. Hillsdale, NJ: Lawrence Erlbaum Associates.

Borod, J.C., Koff, E., Lorch, M. y Nicholas, M. (1985). Channels of emotional expression in patients with unilateral brain damage. Archives of Neurology, 42(4), 345-348.

Borod. J.C., Koff, E., Lorch, M. y Nicholas, M. (1986a). The expression and perception of facial emotion in brain-damaged patients. Neuropsychologia, 24, 169-180.

Borod, J.C., Koff, E., Lorch. M. y Nicholas, M. (1986b). Deficits in facial expression and movement as a funyon of brain damage. En J.L. Nespoulous, P. Perron y A.R Lecours (eds.), The biological foundations of gestures. Hillsdale, NJ: Lawrence Erlbaum Associates.

Borod, J.C., Koff, E., Lorch, M., Nicholas, M. y Welkowitz, J. (1988). Emotional and non-emotional facial behaviour in patients with unilateral brain damage. Journal of Neurology, Neurosurgery y Psychiatry, 51(6), 826-832.

Borod, J.C., Koff, E. y White, B. (1983). Facial asymmetry in posed and spontaneous expressions of emotion. Brain and Cognition, 2, 165-175.

Borod, J.C., Lorch, M., Koff, E. y Nicholas, M. (1987). Effect of emotional context on bucco-facial apraxia. Journal of Clinical and Experimental Neuropsycho$\log y, 9(2), 155-161$.

Cahill, L.F., Uncapher, M.R., Kilpatrick, L., Alkire, M.T., Turner, J. (2004). Sex-related hemispheric lateralization of amygdala function in emotionallyinfluenced memory: An fMRI investigation. Learning y Memory, 11(3), 261266.

Calvo, M.G. y Castillo, M.D. (2005). Foveal vs. parafoveal attention-grabbing power of threat-related information. Experimental Psychology, 52, 150-162.

Calvo, M.G. y Eysenck, M.W. (2008). Affective significance enhances covert at- 
tention: roles of anxiety and word familiarity. QJ Exp Psychol (Colchester), 61, 1669-1686.

Calvo, M.G., Castillo, M.D. y Fuentes, L.J. (2006). Processing of "unattended" threat-related information: Role of emotional content and context. Cognition and Emotion, 20, 1049-1075.

Canli, T., Desmond, J.E, Zhao, Z., Glover, G. y Gabrieli, D.E. (1998). Hemispheric asymmetry for emotional stimuli detected with fMRI. NeuroReport, 9, 3233-3239.

Carstensen, L.L. y Charles, S.T. (1994). The salience of emotion across the adult life span. Psychol. Aging, 9(2), 259-64.

Carstensen, L.L. y Charles, S.T. (1998). Emotion in the second half of life. Curr. Dir. Psychol. Sci., 7(5): 144-49.

Cicero, B.A., Borod, J.C., Santschi, C., Erhan, H.M., Obler, L.K., Agosti, R.M., et al. (1999). Emotional versus non-emotional lexical perception in patients with right and left brain damage. Neuropsychology, and Behavioral Neurology, $12,255-264$.

Dewaele, J.M. (2004a). The emotional force of swearwords and taboo words in the speech of multilinguals. Journal of Multilingual and Multicultural Development, 25(2-3), 204-223.

Dewaele, J.M. (2004b). Blistering barnacles! What language do multilinguals swear in?! Estudios de Sociolingüistica, 5, 83-106.

Dewaele, J.M. (2006). Expressing anger in multiple languages. En A. Pavlenko (ed.), Bilingual minds: Emotional experience, expression, and representation (pp. 118-151). Clevedon, UK : Multilingual Matters.

Dewaele, J.M. (2008). The emotional weight of "I love you" in multilinguals' languages. Journal of Pragmatics, 40, 1753-1780.

Duda, P. y Brown, J. (1984). Lateral asymmetry of positive and negative emotions. Cortex, 20, 253-261.

Fancher, R.E. (1990). Pioneers of Psychology, 2a ed. New York: W.W. Norton \& Co.

Ferré, P., García, T., Fraga, I., Sánchez-Casas, R. y Molero, M. (2010). Memory for emotional words in bilinguals: Do words have the same emotional intensity in the first and in the second language? Cognition and Emotion, 24, 760-785.

Friederici, A.D. (1997). Neurophysiological aspects of language processing. Clinical Neuroscience, 4, 64-72.

Fuentes, L.J., y Santiago, E. (1999). Spatial and semantic inhibitory processing in schizophrenia. Neuropsychology, 13, 259-270.

Goldstein, K. (1939). The Organism. New York: American Books.

González-Reigosa, F. (1976). The anxiety arousing effect of taboo words in bilinguals. En C.D. Spielberger y R. Díaz-Guerrero (eds.), Cross-cultural anxiety (pp. 89-105). Washington, DC: Hemisphere. 
Goodglass, H., Graves, R. y Landis, T. (1980). Le rôle de l'hémisphère droit dans la lecture. Revue Neurologique, 136, 669-673.

Graves, R., Landis, T. y Goodglass, H. (1981). Laterality and sex differences for visual recognition of emotional and non-emotional words. Neuropsychologia, 19, 95-102.

Gross, J.J., Carstensen, L.L., Patsupathi, M., Tsai, J., Skorpen, C.G. y Hsu, A. (1997). Emotion and aging: Experience, expression and control. Psychology and Aging, 12(4), 590-599.

Hamann, S. (2001). Cognitive and neural mechanisms of emotional memory. Trends in Cognitive Sciences, 5, 394-400.

Harris, C.R. y Pashler, H.E. (2004). Attention and the processing of emotional words and names: Not so special after all. Psychological Science, 15, 171-178.

Hielscher, M. (2004). Comprehension of emotional information in patients with aphasia. Folia Phoniatr Logop, 56, 14-26.

Hirschman, R.S. y Safer, M.A. (1982). Hemisphere differences in perceiving positive and negative emotions. Cortex: A Journal Devoted to the Study of the Nervous System and Behavior, 18(4), 569-580.

Holmes, A., Vuilleumier, P. y Eimer, M. (2003). The processing of emotional facial expression is gated by spatial attention: evidence from event-related potentials. Cognitive Brain Research, 16, 174-184.

Kutas, M. y Hillyard, S.A. (1980). Reading senseless sentences: Brain potentials reflect semantic incongruity. Science, 207, 203-205.

Landis, T. (2006). Emotional words: What's so different from just words? Cortex, 42(6), 823-30.

Landis, T., Graves, R. y Goodglass, H. (1982). Aphasic reading and writing: Possible evidence for right hemisphere participation. Cortex, 18, 105-112.

Landis, T., Regard, M., Graves, R. y Goodglass, H. (1983). Semantic paralexia: A release of right hemisphere function from left hemispheric control? Neuropsychologia, 21, 359-364.

Langley, L.K., Rokke, P.D., Stark, A.C., Saville, A.L., Allen, J.L. y Bagne, A.G. (2008). The emotional blink: Adult age differences in visual attention to emotional information. Psychology and Aging, 23 (4), 873-885.

Lavie, N. y Fox, E. (2000). The rol of perceptual load in negative priming. Journal of Experimental Psychology. Human Perception and performance, 26, 1038-1052.

Lavric, D., Pizzagalli, S., Forstmeier y Rippon, G. (2001). Mapping dissociations in verb morphology. Trends in Cognitive Science, 5, 301-308.

Lawton, M.P., Kleban, M.H., Rajagopal, D. y Dean, J. (1992). Dimensions of affective experience in three age groups. Psychology and Aging, 7, 171-184.

Lesser y Milroy (1993). Linguistic and aphasia: Psycholinguistic and pragmatic aspects of intervention. Harlow: Longmans.

Lorch, M.P., Borod, J.C. y Koff, E. (1998). The role of emotion in the linguis- 
tic and pragmatic aspects of aphasic performance. Journal of Neurolinguistics, 11(1-2), 103-118.

MacLeord, C.M., Chiappe, D.L. y Fox, E. (2002). The crucial roles of stimulus matching and stimulus identity in negative priming. Psychonomic Bulletin and Review, 9, 521-528.

McManis, M.H., Bradley, M.M., Berg, W.K., Cuthbert, B.N. y Lang, P.J. (2001). Emotional reactivity in children: Verbal, physiological, and behavioral responses to affective pictures. Psychophysiology, 38, 222-231.

Mohr, C., Michel, C.M., Lantz, G., Ortigue, S., Viaud-Delmon, I. y Landis, T. (2005). Brain state dependent functional hemispheric specialization in men but not women. Cerebral Cortex, 15, 1451-1458.

Mondor, T.A. y Bryden, M.P. (1992). On the relation between visual spatial attention and visual field asymmetries. Quarterly Journal of Experimental Psychology: Human Perception and Performance, 44A, 529-555.

Neshat-Doost, H., Moradi, A., Taghavi, R., Yule, W. y Dalgleish, T. (1999). The development of a corpus of emotional words produced by children and adolescents. Personality and Individual Differences, 27, 433-451.

Ortells, J.J., Tudela, P., Noguera, C. y Abad, M.J. (1998). Attentional orienting within the visual field in a lexical decision task. Journal of Experimental Psychology: Human Perception and Performance, 24, 1675-1689.

Ortigue, S., Michel, C., Murray, M., Mohr, C., Carbonnel, S. y Landis, T. (2004). Electrical neuroimaging reveals early generator modulation to emotional words. NeuroImage, 21, 1242-1251.

Pizagalli, D., Regards, M., y Lehmann, D. (1999). Rapid emotional face processing in the human right and left brain hemispheres: An ERP study. Neuroreport, 10, 2691-2698.

Pourtois, G., Grandjean, D., Sander, D., Vuilleumier, P. (2004). Electrophysiological correlates of rapid spatial orienting towards fearful faces. Cereb Cortex, 14, 619-633.

Pulvermüller, F. (2001). Brain reflections of words and their meaning. Trends in Cognitive Science, 5, 517-524.

Rayner, K. (1998). Eye movements in reading and information processing: 20 years of research. Psychological Bulletin, 124, 372-422.

Robinson, M.D. (1998). Running from William James' bear: A review of preattentive mechanisms and their contribution to emotional experience. Cognition and Emotion, 12, 667-696.

Robinson, J.R., Arndt, S.V, Starkstein, S.E, Forrester, A.W. y Geisler, F. (1993). Depression following traumatic brain injury: A 1 year longitudinal study. Journal of Affective Disorders, 27(4), 233-243.

Ross, E. D., Homan R. W. y Buck, R. (1994). Differential hemispheric lateralization of primary social emotions: Implications for developing a comprehensive 
neurology for emotions, repression, and the subconscious. Neuropsychiatry, Neuropsychology, and Behavioural Neurology, 7, 1-19.

Schirmer, A., Kotz, S. y Friederici, A. (2002). Sex differentiates the role of emotional prosody during word processing. Cognitive Brain Research, 14(2), 228-233.

Semenza, C., Pasini, M., Zettin, M., Tonin, P. y Portolan, P. (1986). Right hemisphere patent's judgements on emotions. Act Neurol. Scand, 74, 43-50.

Shaywitz, B. y Shaywitz, S. (1995). Sex differences in the functional organization of the brain for language. Nature, 373, 607-609.

Strauss, E. (1983). Perception of emotional words. Neuropsychologia, 21, 99-103.

Vasa, R.A., Carlino, A.R., London, K. y Min, C. (2006). Valence ratings of emotional and non-emotional words in children. Personality and Individual Differences, 41, 1169-1180.

Vasey, M., el-Hag, N. y Daleiden, E. (1996). Anxiety and the processing of emotionally threatening stimuli: Distinctive patterns of selective attention among high- and low-test-anxious children. Child Development, 67(3), 1173-1185.

Vö, M.L., Jacobs, A.M. y Conrad, M. (2006). Cross-validating the Berlin Affective Word List. Behavioral Research Methods, 38, 606-609.

Vuilleumier, P., Richardson, M.P., Armony, J.L., Driver, J., y Dolan, R. (2004). Distant influences of amygdala lesion on visual cortical activation during emotional face processing. Nature Neuroscience, 7, 1271-1278.

Wager, T., Phan, K.L., Liberzon, I. y Taylor, S. (2003). Valence, gender, and lateralization of functional brain anatomy in emotion: a meta-analysis of finding from neuroimaging. NeuroImage, 19, 513-531.

Zaidel, E. (1983). Disconnection syndrome as a model for laterality affects in the normal brain. En J. Hellige (ed.), Cerebral hemisphere asymmetry: Method, theory, and application (pp. 95-151). New York: Praeger. 